\title{
Outer layers determine the parallel critical field of a superconducting multilayer
}

\author{
W. Maj* and J. Aarts \\ Kamerlingh Onnes Laboratory, Leiden University, P.O. Box 9506, 2300 RA Leiden, The Netherlands
}

(Received 8 April 1991)

\begin{abstract}
It is shown that the behavior of the parallel critical field for superconducting multilayers with two superconducting components is crucially determined by the outer layers. If the outer layers sustain surface superconductivity not only the three-dimensional (3D) behavior at low temperatures and high fields, but, more importantly, the crossover temperature from 2D to 3D behavior is changed. This phenomenon falls outside the Takahashi-Tachiki theory for such multilayers, but a qualitative explanation is given. Also, if no surface superconductivity occurs, the critical field in the 2D region is due to the outer layers and is different from that of the inside layers.
\end{abstract}

\section{INTRODUCTION}

When both sublayers in a metallic multilayer are superconductors, the interplay of different critical temperatures and different coherence lengths can lead to quite anomalous behavior of the critical field $B_{c 2} \|$ parallel to the layers. A vivid example of this was given by Takahashi and Tachiki. ${ }^{1,2}$ They predicted transitions in $B_{c 2 \|}(T)$ with decreasing temperature, from average three-dimensional (3D), to two-dimensional (2D), to "single layer" 3D behavior when both sublayers have the same $T_{c}$ but very different values for their diffusion constants, or coherence lengths. Here, 3D or 2D behavior means that $B_{c 2} \|$ is proportional to $\left(1-T / T_{c}\right)$ or $\left(1-T / T_{c}\right)^{1 / 2}$. The prediction was borne out by experiments on multilayers of $\mathrm{Nb} / \mathrm{NbTi}$ (Ref. 3) and $\mathrm{Nb} / \mathrm{NbZr}{ }^{4-6}$ In such systems, $T_{c}$ of both sublayers is about $10 \mathrm{~K}$, but the value of $d B_{c 2 \perp} / d T$ (or the diffusion constant) typically differs by a factor 10 .

Although qualitatively all experiments show the same behavior, they differ in some important details. Especially from the data on $\mathrm{Nb} / \mathrm{NbZr}$ multilayers it was shown in Ref. 5 that the temperature $T_{2 \mathrm{D}-3 \mathrm{D}}$, where the transition from $2 D$ to $3 D$ behavior takes place, can be found from the simple relation $\xi_{\mathrm{av}}\left(T_{2 \mathrm{D}-3 \mathrm{D}}\right)=\Lambda / 2$. Here, $\xi_{\mathrm{av}}$ is the average (and temperature dependent) coherence length of the multilayer as can be determined from the linear field dependence of $B_{c 2 \perp}$ near $T_{c}$, and $\Lambda$ is the multilayer periodicity. Some such scaling appears reasonable since the transition should occur when the order parameter starts to nucleate in the $\mathrm{NbZr}$, rather than in the $\mathrm{Nb}$ layer, and this cannot happen before the averaged coherence length becomes a fraction of the multilayer wavelength. Analyzing the data of Refs. 3 and 4, however, indicates a transition around $\xi_{\mathrm{av}}=\Lambda / 4$, meaning that those (equivalent) multilayers show a 2D-3D transition at a considerably lower temperature.

At this point it should be remarked that there is one important difference between the multilayers of Ref. 5 and the multilayers of Refs. 3 and 4: the outer layers in the first case consisted of $\mathrm{NbZr}$, in the last case they were $\mathrm{Nb}$. If the outer layers are $\mathrm{NbZr}$, surface superconductivity can be expected both in the $\overline{3 \mathrm{D}}$ "averaged" region near $T_{c}$ and in the 3D "NbZr" region at high fields. This leads to a larger slope (maximally a factor 1.7 ) in both regions, ${ }^{5}$ but it is not obvious that it would lead to a (very) different
$T_{2 D-3 D}$. From a theoretical point of view it should be noted that surface superconductivity is not taken into account in the Takahashi-Tachiki theory and possible consequences have therefore not been calculated.

The differences in $B_{c 2} \|$ for different outer layers touch upon a broader question: which is the archetypal multilayer described by the theory? It can be anticipated that having only $\mathrm{Nb}$ outer layers in order to suppress surface superconductivity will not be enough. If in the 2D regime the $\mathrm{Nb}$ layers may be described as decoupled superconducting layers, it seems reasonable that the transition to superconductivity in that regime is due to the layer which produces the highest critical field. We will show below that for a variety of reasons these layers are precisely the outer layers.

The aim of this paper is to investigate in detail the role of the surface layers in the critical field behavior for both $\mathrm{NbZr}$ and $\mathrm{Nb}$ top and bottom layers. It will then become clear what kind of multilayer should be used for a relevant comparison with the theory. To this end we prepared a set of multilayers of $\mathrm{Nb} / \mathrm{NbZr}$ with equal periodicities, but with outer layers of either $\mathrm{Nb}$ or $\mathrm{NbZr}$ in a range of thicknesses. Results and discussion are given below.

\section{EXPERIMENTAL RESULTS}

The multilayers were prepared by magnetron sputtering in a UHV system with a base pressure of $10^{-9} \mathrm{mbar}$ in an Ar pressure of $5 \times 10^{-3}$ mbar. Separate $\mathrm{Nb}$ and $\mathrm{Zr}$ sources were used and the multilayers were prepared by shuttering the $\mathrm{Zr}$ source. Sapphire substrates were used which were kept near room temperature during deposition in order to minimize the possible effects of interdiffusion. Sputtering rates were continuously measured with a quartz (oscillating crystal) thickness monitor, which was calibrated by Rutherford backscattering (RBS) measurements on single films of $\mathrm{Nb}$ and $\mathrm{NbZr}$. The rates varied less than $5 \%$ for the preparation of a sample and from sample to sample, which is sufficient for the rather thick layers used in our investigations. The composition of the $\mathrm{NbZr}$ layers was checked by electron microprobe and found to be 55 -at. $\% \mathrm{Nb}$ and 45 -at. $\% \mathrm{Zr}$, without appreciable variation over the length of the film and in agreement with RBS results. RBS could also confirm the multilayer character of samples prepared in this way, al- 
though a precise thickness determination of individual layers was hindered by the interfering intensities of the almost equal masses of $\mathrm{Nb}$ and $\mathrm{Zr}$. Moreover, the multilayer structure was confirmed by $\mathrm{x}$-ray measurements on samples with wavelength of about $80 \AA$ (smaller than used below) which showed clear satellites around the main Bragg peak. For the transport measurements, a fourpoint geometry was (wet) etched into the samples having a strip $0.15 \mathrm{~mm}$ wide and a length of $5 \mathrm{~mm}$ between the voltage contacts. Critical fields were measured by sweeping the field while keeping the temperature constant, and were defined by $50 \%$ of the resistive transition.

In order to investigate the effects of different outer layers, multilayers were used consisting of 15 single layers. The inside layers consisted of $240-\AA \mathrm{Nb}$ alternating with $165-\AA \mathrm{NbZr}$; this choice of thicknesses, especially for $\mathrm{Nb}$, is optimal for following $T_{2 \mathrm{D}-3 \mathrm{D}}$. The two outer layers were always of the same type and equally thick. Their thickness was varied from equal to the corresponding inner layer thickness to $\frac{3}{4}, \frac{1}{2}$, and $\frac{1}{4}$ of that value. For brevity, we call these samples $\mathrm{Nbl}, \mathrm{Nb} \frac{3}{4}$, etc., for $\mathrm{Nb}$ outer layers and $\mathrm{NZ1}, \mathrm{NZ} \frac{3}{4}$, etc., for $\mathrm{NbZr}$ outer layers. One multilayer was prepared with outer layers of $1200-\AA \mathrm{Nb}(5$ times the inner layers); it is called Nb5. The results are collected in Fig. 1 for samples with $\mathrm{NbZr}$ outer layers, and in Fig. 2 for samples with $\mathrm{Nb}$ outer layers. Sample $\mathrm{Nb} 5$ is shown in both figures. For several samples the data points are replaced by a line through the points in order to avoid confusion. Temperatures are scaled on $T_{c}$ 's; for all samples $T_{c}$ 's were about $10.4 \mathrm{~K}$ and varied less than $0.2 \mathrm{~K}$.

Figure 1 shows conspicuous differences, especially between $\mathrm{NZ1}$ and $\mathrm{Nb1}$ or $\mathrm{Nb5}$. Near $T_{c}$, the difference in slopes $(0.65 \mathrm{~T} / \mathrm{K}$ for $\mathrm{NZ1}$ and $0.4 \mathrm{~T} / \mathrm{K}$ for $\mathrm{Nb} 1, \mathrm{Nb} 5)$ is not very surprising. For $\mathrm{NZ} 1$ the value is larger by a factor 1.7 with respect to the slope in perpendicular field while for $\mathrm{Nb1}, \mathrm{Nb} 5$ the value is the same as in perpendicular field. Surface superconductivity therefore appears to be present in this regime if the outer layers are $\mathrm{NbZr}$, which can be expected. The $2 \mathrm{D}$ region is very similar for the three samples, but the transition temperature $T_{2 \mathrm{D}-3 \mathrm{D}}$

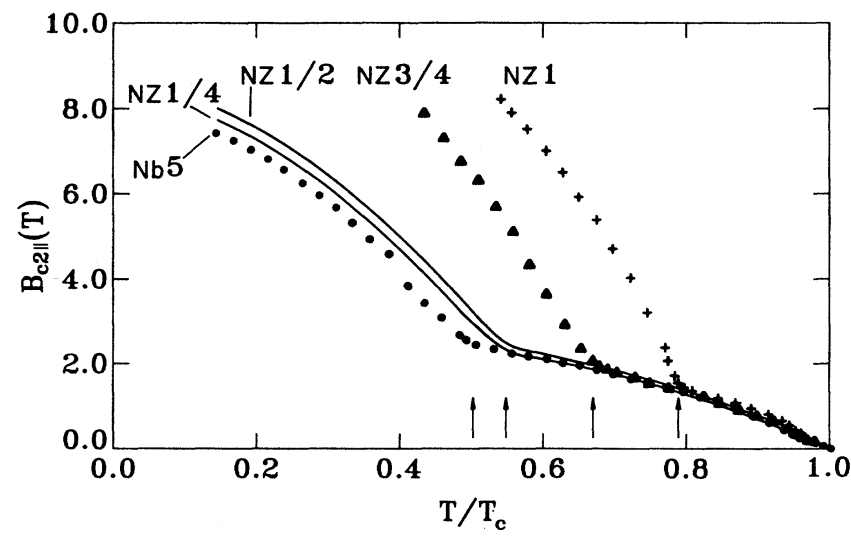

FIG. 1. $B_{c 2 \|}\left(T / T_{c}\right)$ for multilayers with $\mathrm{NbZr}$ outer layers and for $\mathrm{Nb5}$. Lines through points have been drawn for two samples for increased clarity. Arrows denote $T_{2 \mathrm{D}-3 \mathrm{D}} / T_{c}$.

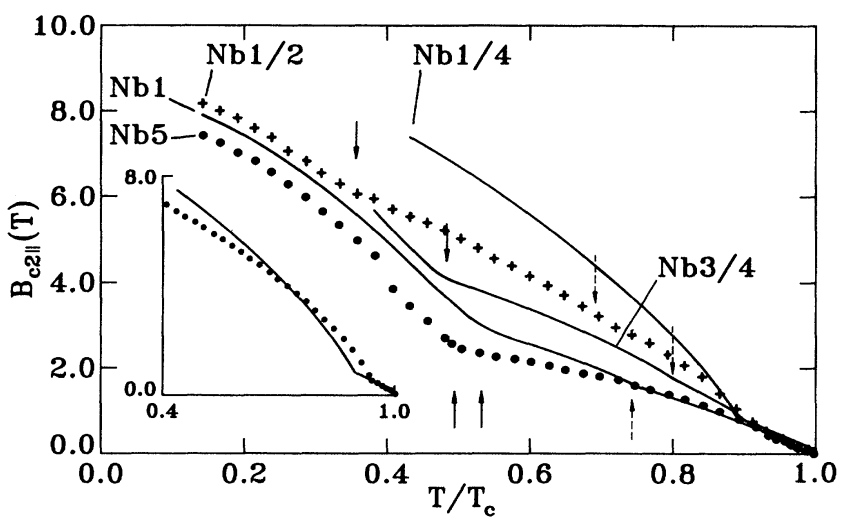

FIG. 2. $B_{c 2 \|}\left(T / T_{c}\right)$ for multilayers with $\mathrm{Nb}$ outer layers. Lines through points have been drawn for three samples for increased clarity. Solid arrows denote $T_{2 \mathrm{D}-3 \mathrm{D}} / T_{c}$, dotted arrows show kinks in the 2D region (see text). The inset shows $\mathrm{Nb} \frac{1}{4}$ (solid line) and the sample with a $\frac{1}{4} \mathrm{Nb}$ buried layer (points).

is reached at a much higher temperature for NZ1. Also the asymptotic slope at high fields is different, $1.7 \mathrm{~T} / \mathrm{K}$ for $\mathrm{NZ1}$ compared to $1 \mathrm{~T} / \mathrm{K}$ for $\mathrm{Nb} 1, \mathrm{Nb} 5 .^{7}$ These observations clearly answer one question raised in the introduction: a drastic change in behavior is witnessed upon changing the outer layers. Figure 1 also shows how $T_{2 \mathrm{D}-3 \mathrm{D}}$ (shown with arrows) changes when the thickness of the $\mathrm{NbZr}$ outer layers decreases. For $\mathrm{NZ} \frac{3}{4}, T_{2 \mathrm{D}-3 \mathrm{D}}$ has decreased halfway to the $\mathrm{Nb5}$ value; for $\mathrm{NZ} \frac{1}{2}$ the $\mathrm{Nb} 5$ value is essentially reached, while the asymptotic region now is also the same as for Nb5. Note that in the 2D region the curves coincide for all samples up to each $T_{2 \mathrm{D}-3 \mathrm{D}}$.

This is not the case when the thickness of the $\mathrm{Nb}$ outer layers is changed, as shown in Fig. 2. The critical field in the $2 \mathrm{D}$ region increases with decreasing layer thickness and this also affects $T_{2 \mathrm{D}-3 \mathrm{D}}$. The transition is still clearly visible for $\mathrm{Nb} \frac{3}{4}$, while for $\mathrm{Nb} \frac{1}{2}$ it becomes a small effect; for $\mathrm{Nb} \frac{1}{4}$ no transition is present in the measured field range. Also, we find small kinks in the $2 \mathrm{D}$ region, marked with dotted arrows in Fig. 2.

\section{DISCUSSION AND CONCLUSIONS}

The above results show that, when going from a multilayer with $\mathrm{NbZr}$ on the outside to a multilayer with $\mathrm{Nb}$ on the outside, the most significant change takes place in the temperature $T_{2 D-3 D}$ which is the crossover from $2 \mathrm{D}$ to $3 \mathrm{D}$ behavior. $T_{2 D-3 D}$ appears to be determined by the final state of the system at low temperatures and is fundamentally higher when surface superconductivity occurs. Before we can discuss this phenomenon it is necessary to reiterate, briefly, the physical reasons for the occurrence of two dimensional crossovers in these multilayer systems and to parametrize the above behavior. At the first ( $\overline{3 D}$ 2D) crossover the superconducting order parameter starts to nucleate in the $\mathrm{Nb}$ layers preferentially. Since the coherence length of the $\mathrm{Nb}$ layers is larger than that of the enclosing $\mathrm{NbZr}$ layers the order parameter is more or less confined and the layer behaves like a thin film with a 
critical temperature $T_{c 2 \mathrm{D}}$ and enhanced critical field

$$
B_{c 2 \|}=\sqrt{12} \frac{\phi_{0}}{2 \pi} \frac{1}{d_{\mathrm{eff}} \xi_{\mathrm{eff}}(0)}\left(1-T / T_{c 2 \mathrm{D}}\right)^{1 / 2} .
$$

This is the standard formula for the behavior of thin films, where $d_{\text {eff }}$ and $\xi_{\text {eff }}(0)$ are the effective thickness and coherence length of the $\mathrm{Nb}$ layer. These differ slightly from the bare $\mathrm{Nb}$ values [ $d_{\text {eff }}$ larger, $\xi_{\text {eff }}(0)$ smaller] because of proximity coupling to the $\mathrm{NbZr}{ }^{5}$ If the order parameter were to nucleate in the $\mathrm{NbZr}$ layers, it would not be confined, but would be spread out and this would just result in a continuation of the $\overline{3 D}$ regime. At the second (2D-3D) crossover, $\xi_{\mathrm{av}}(T)$ has decreased so much that nucleation in the $\mathrm{NbZr}$ layers leads to the bulk critical field of $\mathrm{NbZr}$, without much averaging over other layers involved. $T_{2 D-3 D}$ is therefore the temperature at which averaged behavior changes to bulk $\mathrm{NbZr}$ behavior, and it seems reasonable that this should occur when $\xi_{\text {av }}\left(T_{2 \mathrm{D}-3 \mathrm{D}}\right)$ becomes a fraction $f$ of the multilayer periodicity $\Lambda$. In the case of $\mathrm{NZ1}$ we estimate $\xi_{\mathrm{av}}\left(T_{2 \mathrm{D}-3 \mathrm{D}}\right)=202 \AA$ at $T_{2 \mathrm{D}-3 \mathrm{D}} / T_{c}=0.79$, using the slope of $B_{c 2 \perp}$ near $T_{c}(0.4$ $\mathrm{T} / \mathrm{K})$. This leads to $f=0.5$. In Ref. 5 we used the slope of $B_{c 2 \|}$ for estimating fractions $f$, but this is not quite correct since surface superconductivity occurs in the $\overline{3 D}$ region. Recalculating those results, we find fractions $f \approx 0.5$ for all samples. For sample $\mathrm{Nb} 5$ we find a slope of $0.4 \mathrm{~T} / \mathrm{K}$, $T_{2 \mathrm{D}-3 \mathrm{D}} / T_{c}=0.50, \xi_{\mathrm{av}}\left(T_{2 \mathrm{D}-3 \mathrm{D}}\right)=117 \AA$, and $f=0.29$. This is in agreement with what we inferred from the data on $\mathrm{Nb} / \mathrm{NbTi}$ of Ref. 3 and it remains to explain these different values for $f$. The Takahashi-Tachiki theory ${ }^{1,2}$ cannot help since it does not allow for surface superconductivity, but we may use the following analogy.

Consider the well-known problem of the parallel nucleation field in a slab of thickness $d$ and coherence length $\xi(T)$. Following Saint-James, Thomas, and Sarma, ${ }^{8}$ this field is found for any thickness by solving the linearized Ginzburg-Landau (GL) equation, which we write as

$$
\hbar\left(\nabla / i-2 \pi \mathbf{A} / \phi_{0}\right)^{2} \psi=-2 m a / \hbar^{2} \psi=\psi / \xi(T)^{2},
$$

where $\mathbf{A}$ is the vector potential, $\phi_{0}$ is the flux quantum, and $a$ is the linear coefficient in the GL equation. With the surface of the sample in the $y, z$ plane at $x=-d / 2$ and the field in the $z$ direction, the gauge of the magnetic vector potential is chosen as $A_{y}=H x, A_{x}=A_{z}=0$, and a solution is tried of the form

$$
\psi=\exp (i k z) g(x) \text {. }
$$

This reduces Eq. (2) to

$$
d^{2} g / d x^{2}+(2 \pi H) / \phi_{0}\left(x-x_{0}\right)^{2} g=g / \xi(T)^{2},
$$

where we used $x_{0}=\phi_{0} /(2 \pi H) k$.

Equation (4) now has to be solved with boundary conditions $d g / d x=0$ at $x=-d / 2, d / 2$. A transformation of coordinates $t=\left(4 \pi H / \phi_{0}\right)^{1 / 2} x$ brings Eq. (4) in the wellknown form of a dimensionless eigenvalue equation:

$$
d^{2} g / d t^{2}+1 / 4\left(t-t_{0}\right)^{2} g=\varepsilon g .
$$

Here, $\varepsilon$ is defined as $\varepsilon=\phi_{0} / 4 \pi H \xi(t)^{2}$, or equivalently as $\varepsilon=H_{c 2 \perp} / 2 H$, which gives the relation between the eigenvalue $\varepsilon$ and the corresponding critical field $H$. The field $H$ depends on $x_{0}$ (or $t_{0}$ ), which can be thought of as the nucleation point for a superconducting sheet.

The value $t_{0}$ should be optimized for the lowest $\varepsilon$ for a given value of $d$. If the sample is thick, the bulk solution is found for $t_{0}, x_{0}$ far from the sample surface and corresponds to $\varepsilon=\frac{1}{2}$. The lowest eigenvalue, however, can be obtained for $t_{0}=1.09$ and has a value $\varepsilon=0.293$, which leads to $H / H_{c 2 \perp}=1.7$, the enhancement factor for the surface superconducting critical field. If the sample is thin, the lowest $\varepsilon$ is always found by choosing $t_{0}=0$ (in the middle of the slab). The dependence of $\varepsilon$ on $d / \xi(T)$ is given in Fig. 3 for two cases. In the first case (solid line), $t_{0}$ is optimized for every thickness. A sharp crossover in functional dependence occurs at $d_{c}=1.84 \xi(T)$, which signifies the crossover from thin-film behavior to surface superconductivity. ${ }^{9}$ The second case (dotted line) is when surface superconductivity is suppressed, for instance by cladding the film with a normal metal. This can be mimicked by fixing $t_{0}$ at 0 , giving the crossover from thin-film behavior to bulk superconductivity. As shown in Fig. 3, this crossover is less sharp and occurs around $d_{c} / \xi(T)$ $=3-4$, or at a rather larger thickness than the crossover to surface superconductivity.

We now assert that the following analogy can be made. Both thin-film behavior $(\xi \gg d)$ and averaged behavior in the multilayer $\left(\xi_{\text {av }} \gg \Lambda\right)$ is characterized by an order parameter which is constant over the film and for the multilayer is at least spread out over more layers. For a thin film, crossover to surface superconductivity is around $d_{c}=2 \xi$. For a multilayer, crossover to surface superconductivity is around $\Lambda=2 \xi_{\mathrm{av}}$ (or $\xi_{\mathrm{av}} / \Lambda=0.5$ ), where $\Lambda$ is the wavelength of the outer bilayer because that is where the surface superconductivity will nucleate. This has been found repeatedly. Crossover to bulk superconductivity, however, will be around $\Lambda=3-4 \xi_{\mathrm{av}}$, or $\xi_{\mathrm{av}} / \Lambda=0.3-0.25$, as is found for $\mathrm{Nb5}$. An interesting point is that at the crossover the coherence length is changing from the larger $\xi_{\text {av }}$ to the smaller $\xi_{\mathrm{NbZr}}$, which may accelerate the crossing. For smaller thicknesses of the outer $\mathrm{NbZr}$ it is still the outer bilayer which determines the crossover. For $\mathrm{NZ} \frac{3}{4}$ there is surface superconductivity and the data yield $f=0.45$ when $\Lambda$ is taken as $365 \AA$, the thickness of

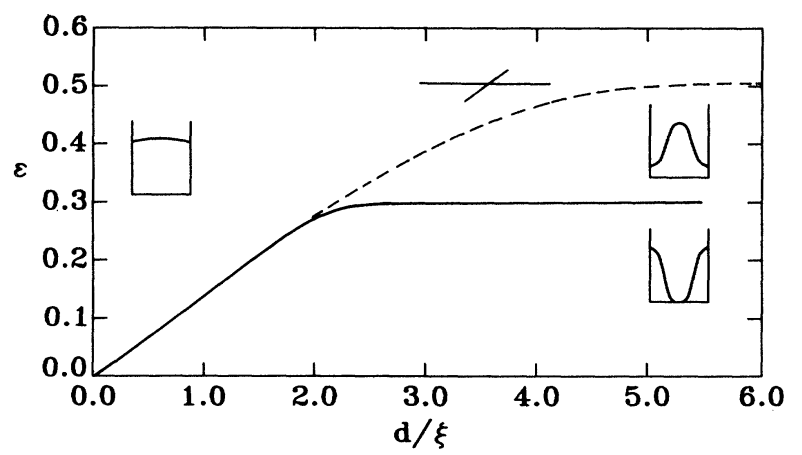

FIG. 3. Eigenvalues $\varepsilon$ as function of $d / \xi$ from Eq. (5) for the cases when $t_{0}$ is optimized (solid line) or fixed to 0 (dotted line). The region for crossover from thin film to bulk behavior is indicated, and a schematic picture of the order parameter in different regions is given. 
the outer bilayer. For NZ $\frac{1}{2}$ and $N Z \frac{1}{4}$ the data are very similar to $\mathrm{Nb} 5$, indicating that the thin $\mathrm{NbZr}$ layer does not sustain surface superconductivity. The crossover, therefore, is to nucleation in the inner $\mathrm{NbZr}$ layers for which the full multilayer $\Lambda$ is decisive. This yields $\xi / \Lambda$ $=0.32$ for both samples $\mathrm{NZ} \frac{1}{2}$ and $\mathrm{NZ} \frac{1}{4}$.

The situation with varying outer $\mathrm{Nb}$ thickness is simpler. The data show unequivocally that the critical field in the $2 \mathrm{D}$ region is determined by the thickness of the outer layers only. Following Eq. (1) this results in a steady increase of the field in this region with decreasing thickness. $T_{2 D-3 D}$ occurs at the crossing with the underlying multilayer behavior (the curved asymptotic regime). This is most strikingly seen in the data for $\mathrm{Nb} \frac{3}{4}$ and $\mathrm{Nb} \frac{1}{2}$ : the former is already in the asymptotic multilayer regime, while $T_{2 \mathrm{D}-3 \mathrm{D}}$ for the latter occurs exactly where the data coincide. No simple rule can therefore be given for $T_{2 \mathrm{D}-3 \mathrm{D}}$ in this situation.

These experiments show that for not only $\mathrm{NbZr}$ but also for $\mathrm{Nb}$ outer layers care has to be taken when comparing with theory. First, if the top layer is not protected, it may oxidize and thereby become effectively thinner; as our experiments show, this will enhance the critical field in the $2 \mathrm{D}$ region. There may even be a difference between the top and bottom layer, which we think is the explanation for the observed kinks. Especially for sample Nbl (Fig. 2) it appears that below $t=0.75$ the field is determined by a layer of smaller thickness and a slightly lower $T_{c 2 \mathrm{D}}$, due to a disordered or oxidized $\mathrm{Nb}$ layer. Obviously, this problem is more stringent for thinner layers. The second reason is more intrinsic. It was shown in Ref. 5 that the thickness of the layer causing 2D behavior was the thickness of the $\mathrm{Nb}$ layer plus a fraction of the (proximity-coupled) $\mathrm{NbZr}$ on each side. The outer layers, with only $\mathrm{NbZr}$ on one side, are therefore effectively thinner than the inner layers. Again this causes a higher critical field while also a smaller "dressing" should be found for multilayers with $\mathrm{Nb}$ on the outside. An illustration of these points is given by measurements on a special sample which has one thin layer $\mathrm{Nb}$ buried inside the multilayer: it consists of three double-layers $\mathrm{Nb} / \mathrm{NbZr}$, one $\frac{1}{4}$-layer $\mathrm{Nb}$, and three double-layers $\mathrm{NbZr} / \mathrm{Nb}$. The data are shown in the inset of Fig. 2, together with the data on $\mathrm{Nb} \frac{1}{4}$. The measurements are almost the same, but the buried layer shows a slightly higher $T_{c 2 \mathrm{D}}$ (better proximity coupling with $\mathrm{NbZr}$ ) and a slightly lower $B_{c 2 \|}$ at low temperatures (the inner layer is effectively thicker). Of course, it should not be a surprise anymore that the one buried layer, and not the rest of the multilayer, completely determines the critical field in the $2 \mathrm{D}$ region. Generally speaking, this also contains a warning against the (symmetry) argument that a multilayer should be terminated with layers of half the inner thickness.

As a final remark, our experiments show that in resistive measurements on the above class of multilayers often only the superconducting transition of parts of the multilayer is observed. This implies that below $B_{c 2}$ a kind of phase diagram exists, which can actually be probed by measuring the critical current below $B_{c 2}$. The result of these measurements will be reported elsewhere.

\section{ACKNOWLEDGMENTS}

We would like to acknowledge useful and stimulating discussions with K.-J. de Korver, P. Koorevaar, P. H. Kes, and $\mathbf{J}$. Mydosh. We are grateful to the Institute for Atomic and Molecular Physics (AMOLF) for help with the RBS measurements. This work is part of the research program of the Dutch Foundation for Fundamental Research on Matter (FOM).
*Permanent address: Institute of Physics, Polish Academy of Sciences, Al. Lotnikow 32/46, 02-668 Warsaw, Poland.

${ }^{1}$ S. Takahashi and M. Tachiki, Phys. Rev. B 33, 4620 (1986).

${ }^{2}$ S. Takahashi and M. Tachiki, Phys. Rev. B 34, 3162 (1986).

${ }^{3}$ M. G. Karkut, V. Matijasevic, L. Antognazza, J.-M. Triscone, N. Missert, M. R. Beasly, and $\emptyset$. Fischer, Phys. Rev. Lett. 60, 1751 (1988).

${ }^{4}$ Y. Kuwasawa, U. Hayano, T. Tosaka, S. Nakano, and S. Matuda, Physica C 165, 173 (1990).

${ }^{5}$ J. Aarts, K.-J. de Korver, and P. H. Kes, Europhys. Lett. 12,
447 (1990)

${ }^{6}$ J. Aarts, K.-J. de Korver, W. Maj, and P. H. Kes, Physica B $165 \& 166,475$ (1990).

${ }^{7}$ At these low temperatures the asymptotic behavior is actually not linear anymore, but curved in accordance with the Werthamer-Helfand-Hohenberg theory.

${ }^{8}$ D. Saint-James, E. J. Thomas, and G. Sarma, in Type II Superconductivity, International Series of Monographs in Natural Philosophy (Pergamon, New York, 1969), Vol. 17.

${ }^{9}$ H. J. Fink, Phys. Rev. 177, 732 (1969). 